


\title{
Mujeres errantes: motivaciones y trayectorias de la migración uitoto a Bogotá
}

\author{
Irene Vélez Torres*
}

\section{Metodología y campo empírico}

Este texto reflexiona sobre la migración como una práctica social vinculada a situaciones simultáneas de carencia socioeconómica y violencia por conflicto armado en los territorios donde tradicionalmente ha vivido el pueblo uitoto. La información empírica que aquí se presenta fue construida a través de una investigación desarrollada entre los años 2005 y 2006, la cual se enfocó en reconstruir las trayectorias de movilidad y las prácticas socioculturales urbanas de diez mujeres indígenas que se encontraban viviendo en Bogotá, en su mayoría provenientes de la región amazónica colombiana.

* Candidata a Ph.D. en Geografía y Geología, University of Copenhagen, Ms.A. en Estudios Culturales y BA en Filosofía de la Universidad Nacional de Colombia. Investigadora del Centro de Estudios Social de la Universidad Nacional de Colombia. Ha centrado su trabajo en el análisis de los conflictos socioambientales, la migración, la etnicidad y las luchas de los movimientos sociales en Colombia. Correo electrónico: irenevt@gmail.com 
La mayoría de estas mujeres manifestaron que su migración era forzada (en oposición a una migración voluntaria), bien sea por causas asociadas al conflicto armado en sus territorios, o bien, por causas relacionadas con violaciones a otros derechos fundamentales. Resulta interesante, en este sentido, su autoidentificación como "desplazadas", más allá de la certificación que hubiesen obtenido o no por parte del Estado. Así, en sus relatos, la violencia y el acceso limitado y de baja calidad a servicios básicos eran presentados como motivaciones de migración tan cruciales como los enfrentamientos y "tomas" de sus territorios por parte de actores armados, legales e ilegales. $\mathrm{Al}$ ubicar el conflicto armado en el mismo nivel explicativo que la falta de empleo o de servicios de salud -derechos imprescindibles para garantizar una calidad de vida adecuada y digna-, estas mujeres sugieren un cuestionamiento sobre la complejidad de la violencia y sobre las distinciones tajantes entre los factores que pueden impulsar la migración.

Cuando en el discurso de estas mujeres emerge la violencia de las armas y del empobrecimiento con la misma fuerza, las distinciones entre desplazamiento y otras formas de migración se relativizan, a la vez que surgen toda clase de dudas y ambigüedades sobre las definiciones institucionales y las fronteras, generalmente arbitrarias, que distinguen a los "desplazados" de otros migrantes internos en Colombia. Con estas inquietudes en mente, este ensayo explora las principales motivaciones, prácticas urbanas y trayectorias de movilidad de estas migrantes para mostrar las fronteras difusas de las definiciones y explicaciones monocausales del desplazamiento en Colombia.

En sintonía con Castillejo (2000), quien avanza en una antropología del exilio interno en Colombia a partir del análisis de las condiciones de tránsito de la población desplazada, y no tanto desde su estudio como sujetos de la violencia o de las políticas asistenciales, retomaré a continuación los procesos de migración de estas mujeres menos desde las cifras y más desde las experiencias de su movilidad. Organizaré este texto en dos partes: primero, las motivaciones de la migración; segundo, las formas de producción de 
capitales sociales y económicos en la ciudad. Concluiré que la actual migración de estas mujeres se enmarca en una trayectoria amplia de movilidad que articula diferentes factores de expulsión, siendo el conflicto armado y el empobrecimiento dos caras de una misma monera. Por otro lado, mostraré que las dificultades que enfrentan estas mujeres para vivir dignamente en la ciudad, aunadas a las expectativas de recuperar prácticas cotidianas de libertad en los territorios tradicionales, hacen que la migración (incluso aquella catalogada como "desplazamiento") sea un práctica permanente de entrada y salida, más que un proceso definitivo de abandono territorial y cultural.

\section{Motivaciones}

La experiencia del lugar urbano para las mujeres indígenas migrantes con quienes desarrollé esta investigación se construye en permanente contrapunteo con la vida rural. Estas mujeres narran las prácticas cotidianas en los territorios locales de donde provienen a través de, por un lado, discursos (Foucault, 1983) que oscilan entre la abundancia de la comida, los espacios fisicos y sociales, y las relaciones afectivas; y, por otro lado, de la violación del derecho a la vida, a la salud, a la educación y al trabajo. Su nostalgia reitera la abismal diferencia entre la infraestructura urbana y la precariedad de la vida rural, anclada en la desigualdad social y económica entre los pobladores de las ciudades y aquellos del campo, en relación con su acceso a diferentes servicios propios de la modernidad (García-Canclini, 1990). Sin embargo, ello no quiere decir que la ciudad esté llena de "riquezas" a las cuales puedan acceder las mujeres indígenas que llegan a vivir a Bogotá, pues si bien la vida urbana ofrece unas posibilidades infraestructurales e institucionales que se extrañan en la región amazónica, lo cierto es que estas mujeres tienen pocas oportunidades de acceder a ellas.

Para entender las motivaciones ambivalentes de estas mujeres, expresadas entre acogida-rechazo, abundancia-carencia y libertad-violencia, es 
necesario reconocer, en primer lugar, que históricamente la región amazónica ha estado surcada por diferentes procesos de violencia, asociados a la incesante acumulación de capital por extracción (Harvey, 2007). Los sistemas productivos que han dejado huella en el paisaje amazónico se enmarcan en la economía capitalista global y pueden ser rastreados a través de auges productivos que han coincidido con picos locales de violencia: la quina (Cinchona pubescens y Cinchona parabolica) a finales del siglo XIX (Palacio, 2003), el caucho (Hevea brasiliensis) durante la primer a mitad del siglo XX (Pineda Camacho, 2000), la coca (Cocos nucifera) desde la década de 1970 (Roncken, 2004), el petróleo desde 1990, actualmente algunos servicios ambientales como el turismo, y los bonos de carbono en los mercados voluntarios de reducción de emisiones por deforestación y degradación de los bosques (REDD y REDD+). Estos procesos de ocupación del espacio amazónico para el comercio y la impronta de la violencia que suponen las diferencias entre los intereses del extractivismo y de los pobladores locales han sido factores de expulsión ampliamente documentados.

En segundo lugar, es importante analizar otros factores que han sido complementarios a estos procesos extractivistas y que permiten relacionar la violencia de la extracción con la violencia del empobrecimiento como dos procesos no solo simultáneos, sino esencialmente entrelazados. Así, cuando iniciaba mi investigación, una líder del movimiento indígena me subrayó la importancia de indagar las causas y motivaciones de la migración. Esta líder sugirió algunas distinciones:

Para abordar el tema de los indígenas en la ciudad hay que comenzar diferenciando las razones por las que el indígena puede buscar venir a una ciudad como Bogotá: primero, porque son líderes o activistas [y su] trabajo es nacional y deben desplazarse por todo el territorio, teniendo como centro Bogotá. Segundo, [algunos] indígenas que vienen por un periodo de tiempo buscando educarse y conocer otras cosas. Tercero, indígenas que vienen dejando atrás su ser indígena, para buscar nuevas 
formas de vida, especialmente buscando emplearse, algunas mujeres como empleadas del servicio y prostitutas. [Y otros] indígenas vienen desplazados por la violencia que se vive en las regiones.

Ella refirió estas motivaciones más como factores de expulsión de los territorios tradicionales que como categorías excluyentes de diferentes tipos de migrantes. Entre estos factores, el único que se diferencia cualitativamente de los demás es el de "vivir de otra manera", pues lleva de suyo una aparente renuncia a la identidad étnica y al origen territorial y cultural indígena del migrante. No porque fuera menos común, sino por la sanción moral que le merecía, esta líder restringía sus relaciones sociales a aquellos (as) indígenas cuya motivación tenía que ver con la violencia o con "la falta de posibilidades", criticando y marginando a aquellos para quienes el abandono del territorio tradicional era un deseo.

La ausencia de garantías para el cumplimiento de los derechos humanos y culturales de los habitantes de la región amazónica fue también referida por otra mujer entrevistada, quien además de ser hija de un reconocido chamán uitoto, trabajaba con la Organización Nacional Indígena de Colombia (ONIC). Al preguntarle por las causas de la migración, ella afirmó:

Pues la violencia [...] Pero mucha gente, la mayoría, se desplaza por lo de educación. Porque hasta ahora, solo hasta el año pasado hubo los primeros que salieron de bachiller por allá. Porque la parte de educación está muy abandonada... Entonces los papás se salen junto con los hijos para que entren a la universidad o entren a un colegio. Educación y trabajo van juntos. Porque antes se llegaba a quinto de primaria y ahí se quedaba. Hoy se llega a bachiller, pero ¿qué es un bachiller joven indígena? Pues para ellos no hay nada, no hay empleo, ni condiciones, entonces muchos tiene que salirse. Eso es muy paradójico y entonces muchos pueblos han optado por no ir a las escuelas; ellos quieren más 
bien a sus hijos en la comunidad, que trabajen en lo de ellos; o mandan solo uno o dos que vengan a capacitarse, pero no todos.

El uso del término "desplazado" que se hace aquí refiere a una forma de migración en que las causas han sido ajenas y contrarias a la voluntad del migrante. El desplazamiento es, en este sentido, la migración que se origina en el abandono y que se expresa en las confrontaciones armadas y en la falta de educación y empleo como garantías de una vida digna. A la hora de generalizar, la entrevistada sugiere que la carencia de instituciones educativas en la región amazónica es la principal motivación para la migración, a la vez que señala un mayor desafío para los jóvenes indígenas actualmente: la necesidad que tienen de educarse para acceder al mercado laboral.

El abandono no solo se expresa en la falta de educación y trabajo, sino también en la precariedad o ausencia de servicios de salud con oportunidad y calidad. Varias de las entrevistas giraron en torno a enfermedades personales o de algún familiar que han obligado la migración temporal en busca de los tratamientos médicos adecuados. En varias trayectorias personales de movilidad, estas migraciones temporales para acceder a servicios de salud constituían la primera de una larga cadena de migración, a veces para hacerse a servicios del precario Estado social de derecho en Colombia, a veces huyendo de algún conflicto en el territorio, y la mayoría de las veces por ambas causas simultáneamente. Una de las entrevistadas, rechazando estas condiciones de vida local, criticó al Estado y a las organizaciones indígenas por su falta de atención a estas necesidades:

Hay abandono del Estado, hay inexistencia del Estado y también de las mismas organizaciones indígenas de que los representan a ellos. Ellos están totalmente solos.

El abandono social por parte del Estado invita a cuestionar cuáles son los actores que hacen presencia en la región amazónica, pues la condición de 
triple frontera ha inducido una presencia del Estado restringida al aparato militar, en detrimento de otras instituciones y servicios clave del bienestar de los habitantes locales. Esta militarización legal e ilegal del territorio, en contrapunto con la debilidad en el equipamiento y los servicios que son garantía de derechos, conducen a la pregunta sugerida por varios investigadores sobre la identidad nacional y el Estado-nación en Colombia (CastroGómez, 2003).

En el caso en mención, esta pregunta se ha (de)formado a través de una larga historia de violencias, superpuestas entre sí y arraigadas en el extractivismo. Si bien este cuestionamiento conduce a una indagación distinta de la sostenida en esta investigación, no deberá perderse de vista que las motivaciones de la migración se construyen a través de la constante retroalimentación entre las condiciones de expulsión por conflicto armado y la relación desigual entre la violación de derechos en el territorio y la expectativa de su garantía en las ciudades. De esta manera, los análisis críticos sobre las condiciones de vida territorial, que integra actores e intereses, constituyen importantes aportes en la comprensión de esta dialéctica de la migración: aquella que se genera entre las motivaciones por expulsión y las motivaciones por acogida.

\section{Haciéndose a la vida urbana}

Volviendo al traslape que existe entre las motivaciones de la migración asociadas al conflicto armado y las carencias locales, es interesante mostrar cómo la vida urbana y la distancia física y social con el territorio tradicional va moldeando, en las migrantes, un discurso en que la vida en la ciudad se convierte en una oportunidad para ampliar su conocimiento y, de esta manera, reencontrar su identidad indígena desde una nueva posición de saber-poder (Foucault, 2005). Una mujer joven cuya familia llegó a Bogotá hace varios años, bajo la condición de desplazada, se refirió a su proceso 
académico como una oportunidad de re-encontrar su cultura y reafirmar desde ahí su pertencia identitaria:

A mí la universidad me hizo fue valorar la cultura, como esa pertenencia y identidad. [...] Y en la universidad lo que me di cuenta fue de eso, de que nosotros como pueblos indígenas teníamos mucho conocimiento, una sabiduría muy grande y muy poderosa y que eso lo han intentado opacar. Y todo ese tiempo en la universidad me hizo fue valorar la cultura. La universidad al final se volvió un cartón o un título, pero no era lo que realmente yo quería hacer, no era mi proyecto de vida. Y eso lo que hizo fue más sentir orgullosa de lo que nosotros somos.

En este caso, el desplazamiento se convirtió en una oportunidad para la formación académica y política de esta mujer. Y es precisamente por ello que cuando su familia pudo regresar a la Amazonía un tiempo después de originada la migración, decidió permanecer en la ciudad hasta que la hija hubiese finalizado sus estudios universitarios. Así, se evidencia que antes que segregarse, emerge un reposicionamiento y una articulación estrecha entre los diferentes factores de la migración, indesligables en la experiencia y discernibles solo analíticamente. No quiere decir esto que por aprovechar las oportunidades del contexto urbano se nieguen o silencien las experiencias violentas que esta familia tuvo que afrontar y que les arrojaron a las calles de la urbe, en donde sobreviven vendiendo artesanías y haciendo curaciones en los barrios populares de Bogotá. Lo que me interesa señalar es que hay matices en las motivaciones, y que en la medida en que se cruzan los diferentes factores en la experiencia cotidiana de las migrantes, el mismo proceso de movilidad va tejiendo una madeja densa entre los hilos de la violencia por el conflicto armado y los hilos de otras violencias materiales y simbólicas, relacionadas con el desbalance entre necesidades y carencias.

Todas las mujeres con quienes realicé esta investigación se han esforzado por buscar oportunidades educativas y laborales. Además, han pasado por 
diferentes procesos de educación formal e informal, de los cuales afirman que han sido su principal "ganancia" en la ciudad. Una mujer mayor entrevistada, desempleada y quien había vivido durante nueve años en Bogotá, melancólicamente me dijo:

Pues uno nunca debe de decir que no... Pues sí, sí he ganado..., porque yo no soy la mujer que era allá. Allá era la mujer indígena que no tenía ningún conocimiento, sino únicamente lo que era la cultura nuestra. Pero acá no, acá ya he aprendido mucho, he recibido muchas capacitaciones, he sido mujer ventera en las cooperativas, cosa que yo por allá no hacía eso [...] Pues ya soy una mujer preparada, ya no soy lo que era allá. Ahora ya manejo más, más... ¿cómo se le puede llamar a eso?... Como que yo no sabía cómo tratarme con una persona, a mí como que me daban nervios. Pero ya no, entonces yo digo que como que ya me preparé más.

En esta línea de ideas, Salcedo (2006) ha caracterizado la esperanza de estabilidad y ascenso socioeconómico que los desplazados depositan en los procesos formativos como una práctica urbana clave de los migrantes, quienes protegen con especial esmero los certificados de las capacitaciones realizadas y las cartas de recomendación de antiguos patronos. Y es que innegablemente los procesos de educación en la ciudad permiten la adquisición de un capital social (Chambers, 1995) que las mujeres aprecian y valoran como una oportunidad para "ascender" económicamente. Sin embargo, la melancolía que se revela en este relato consiste precisamente en que, pese a las satisfacciónes que les genera la educación que reciben, las mujeres migrantes enfrentan enormes limitaciones para que el conocimiento adquirido les represente un mejoramiento en sus condiciones de vida. Otro relato que reitera sobre sus frustraciones surgió en una entrevista a una mujer mayor, quien acababa de salir del desempleo a través de la obtención de un trabajo en Misión Bogotá (programa de generación 
de empleo durante la administración de izquierda adelantada por el Polo Democrático Independiente entre 2004 y 2007):

Pero ahorita sí no... Ahorita me desilusioné... De verdad que sí... Pero no mijita, la verdad es que me siento muy mal, yo me siento muy aburrida porque uno hace el esfuerzo de salir adelante o de servir para algo, pero no. Todo lo que yo estudie allá en el SENA y en casa de familia no más. Ahora Misión Bogotá tampoco...

Resulta explícito que las capacitaciones en el SENA no han estado encadenadas a un proceso de inserción laboral, de manera que por más formación que haya recibido, esta mujer solo logra emplearse como doméstica, a la vez que Misión Bogotá constituye un paliativo temporal frente a la oscilación permanente entre desempleo y empleo informal (Portes, 2004). Esta distancia entre las expectativas generadas por el capital social urbano y las oportunidades tangibles de estabilidad y ascenso socioeconómico no solo se expresa en la dificultad que enfrentan estas mujeres para conseguir empleo, sino también en el fracaso de otras estrategias económicas inscritas en la experiencia de la migración.

Así, algunas de las mujeres que han accedido a capacitaciones en el SENA y a subsidios del Estado para la creación de microempresas critican la insuficiencia de esta posibilidad para mejorar su situación económica. Sus relatos revelan la dificultad de consolidar una práctica productiva capaz de garantizar la adquisición de un capital económico suficiente para la supervivencia de cada mujer y su familia. La posibilidad de que esto suceda depende menos del saber técnico y de las garantías suplementarias, y más de las redes sociales y de la experiencia comercial necesarias para atravesar el umbral de la producción hacia la distribución y el consumo de las mercancías producidas.

Desde un análisis cuantitativo de variables de desarrollo económico, Ibáñez y Vélez (2008) también han criticado la efectividad de los programas de 
cooperación internacional para población desplazada que promueven la creación de famiempresas y microempresas, pues no han demostrado que la población beneficiaria gane independencia económica una vez finalizado el periodo de subsidio y apadrinamiento por el programa. Desde una perspectiva más etnográfica, la presente investigación muestra que la carencia de redes sociales y de experiencia comercial es una barrera para que estos programas funcionen como garantía de los derechos económicos de la población migrante. En general, lo que revelan las narraciones de las mujeres entrevistadas es que, al carecer de espacios comerciales apropiados, la venta de los productos "al menudeo" termina por convertir el negocio en "plata de bolsillo", haciéndose muy difícil recuperar el capital inicial y resolviéndose, en última instancia, la venta de las máquinas como recurso para el sostenimiento familiar. Esta situación revela que este tipo de asistencia por parte de las instituciones gubernamentales y de cooperación son insuficientes como alternativa económica para los migrantes, pues mientras no se organicen procesos estables para la comercialización de los productos, los subsidios se desvanecen en cuestión de meses.

Las limitaciones generalizadas para encontrar condiciones de vida satisfactorias en la ciudad se mezclan con otras frustraciones de la vida urbana relacionadas con los nostálgicos recuerdos de las actividades que realizaban en la chagra ${ }^{1}$ y que ya no pueden desarollar en Bogotá:

Es que yo no vivía encerrada, los niños jugaban libremente mientras yo salía a la chagra a sacar la yuca [...] Eso no quisiera yo ni recordar... Cuando me quedo sola en la casa me da mucha tristeza y a veces me pongo a chillar. Antes en mi chagra yo no era así [...] Es que aquí uno vive como más encerrado. Yo no salgo más. Antes iba a mi chagra y allá hacía mis cosas. Aquí no hablo ni con las vecinas [...] Allá vivíamos pobremente pero cómodamente, ¿sí? Tenía yo mi chagra y de ahí nos

1 Espacio femenino para la producción de alimentos de consumo cotidiano, especialmente yuca, plátano y ají. 
producía la comida [...] Antes regalaba y vendía, en cambio acá uno vive como alcanzado.

Estos reclamos y añoranzas permiten una interpretación de la experiencia cotidiana de estas mujeres indígenas en la ciudad: la construcción del lugar urbano se logra a través de prácticas de memoria, donde los espacios y las sustancias (Seremetakis, 1996) dejadas en el territorio tradicional son, antes que olvido, el espacio de representación en el se enmarcan y se dialogan los retos de la vida urbana. En este contexto, cobra especial interés el significado y la magnitud que tiene para las mujeres uitoto no tener una chagra, pues la memoria de este lugar es el racero con el que se piensa y se valora buena parte de la vida en la ciudad.

Así, es necesario reconocer que con la pérdida del espacio-chagra, las mujeres pierden su libertad y su autonomía. Se encuentran ahora obligadas a conseguir trabajos que escasean y a depender de la economía de mercado en que se hallan inmersas. También pierden su movilidad geográfica y social: ya no salen, no hablan con nadie, no comparten los logros que la chagra les permitía. En el hecho de no tener una chagra se debate un asunto que supera la limitación material de no poder cultivar y procesar sus propios alimentos, pues el significado cultural que para las mujeres tiene el trabajo en la chagra se enlaza con el ejercicio del ser femenino, el cual termina por opacarse en sus nuevas actividades urbanas.

\section{Conclusiones}

La adquisición de capitales sociales y económicos en la ciudad es una disputa constante que afrontan las mujeres migrantes, lo cual contribuye a la enorme dificultad que enfrentan para acceder a los servicios y derechos que perseguían en su trayectoria de migración. A esto se suma la nostalgia de estar por fuera del espacio físico y social tradicional, representado, para el caso de las mujeres, en la ausencia de la chagra y la pérdida de 
independencia y, consiguientemente, libertad. La dificultad de la vida urbana le plantea a estas mujeres preguntas constantes sobre las condiciones y oportunidades de retorno; estas reflexiones surgieron en todas las entrevistas realizadas, cuando frente a la pregunta sobre su futuro, la mayoría respondió: "regresar a hacer chagra".

Esta expectativa de volver a la chagra permite pensar en una permanencia cultural en la migración, donde la cotidianidad en el territorio tradicional se revive como un hecho pasado, pero también como una posibilidad de futuro que no se niega ni se olvida en la experiencia urbana. En este contexto, se entiende cómo las dinámicas de "ida y vuelta" son constantes, lo cual refuerza la idea de que existen trayectorias históricas y personales de migración en el pueblo uitoto. Este panorama permite sugerir que la movilidad entre las mujeres no es nueva (Nieto, 2006); en cambio, hace parte de dinámicas e imaginarios de movilidad, arraigados en su memoria y en su cotidianidad.

Ahora bien, que haya trayectorias errantes, personales y comunitarias no implica que las motivaciones y dinámicas de la actual migración estén determinadas a priori, así como tampoco que las dificultades e inequidades en medio de las cuales tiene lugar sean menos injustas. En cambio, lo que se afirma es que la migración ha sido una continuidad histórica en la cual las motivaciones de salida han estado, la mayoría de las veces, asociadas a una trilogía entre violencia armada, extractivismo local y violación de derechos básicos como salud, educación y trabajo. En este sentido, es necesario concluir que las motivaciones de la migración no son monocausales, sino que en ellas se evidencia un reforzamiento perverso entre el conflicto armado, la presión territorial de la economía capitalista y variadas carencias socioeconómicas. De esto se sigue que la distinción entre desplazamiento y migración (no forzada) es, en muchas ocasiones, una diferencia arbitraria, enmarcada más en las complejas prácticas burocráticas del Estado que en las experiencias y narraciones de los migrantes sobre su condición. 


\section{Referencias}

Castillejo, A. (2000). Poética de lo otro: antropología de la guerra, la soledad y el exilio interno en Colombia. Bogotá: Instituto Colombiano de Antropología e Historia - Colciencias.

Castro-Gómez, S. (2003). Latin American philosophy as critical ontology of the present: themes and motifs from a "Critique of Latin American reason". En Mendieta, E. Latin American philosophy: currents, issues, debates. Indiana: University Press.

Chambers, R. (1995). Poverty and livelihoods: whose reality counts? Environment and Urbanization, 1(7).

Escobar, E., Escobar, P., Pazmiño, A. y Ulloa, A. (Eds.) (2005). Las mujeres indígenas en los escenarios de la biodiversidad. Bogotá: Fundación Natura Colombia - Instituto Colombiano de Antropología e Historia.

Foucault, M. (1983). El sujeto y el poder. Recuperado de http://www.campogrupal.com/poder.html

Foucault, M. (2005). Las palabras y las cosas. Una arqueología de las ciencias humanas. México: Siglo XXI.

García-Canclini, N. (1990). Culturas híbridas: estrategias para entrar y salir de la modernidad. México: Grijalbo.

Harvey, D. (2007). El "nuevo" imperialismo. Sobre reajustes espacio-temporales y acumulación mediante desposesión. Buenos Aires: IADE.

Ibáñez, A. M. \& Vélez, C. E. (2008). Civil conflict and forced migration: the microdeterminants and welfare losses of displacement in Colombia. World Development, 4(36), 659-676.

Nieto, J. V. (2006). Mujeres de la abundancia (tesis de grado). Leticia: Universidad Nacional de Colombia. 
Palacio, G. (2003). Corografía, quina y catequización: historia amazónica entre 1850 y 1920. Recuperado de www.estudiosecologistas.org/docs/ reflexion/.../Corografiaquina.pdf

Pineda Camacho, R. (2000). Holocausto en el Amazonas. Una historia social de la Casa Arana. Bogotá: Espasa.

Portes, A. (2004). El desarrollo futuro en América Latina. Neoliberalismo, clases sociales y transnacionalismo. Bogotá: Instituto Latinoamericano para una Sociedad y un Derecho Alternativos.

Roncken, T. (2004). El espiritu guerrerista. La lucha contra las drogas y la protecciónmilitar de los Estados Unidos. Quito: Transnational Institute - Acción Andina Bolivia - Abya-Yala.

Salcedo, A. (2006). Claiming lands from the city: forced displacement and reconstruction in contemporary Colombia. Recuperado de http:// www.humanas.unal.edu.co/colantropos/

Seremetakis, N. (Ed.) (1996). The senses still: perception and memory as material culture in modernity. Chicago: The University of Chicago Press.

Vélez, I. (2006). La inconmensurabilidad conceptual: el caso de la hoja de coca en uitotos y occidente. Inversa, 2(1). Bogotá. 